\title{
Costate Estimation Using Multiple-Interval Pseudospectral Methods
}

\author{
Christopher L. Darby,, , Divya Garg, \\ University of Florida, Gainesville, Florida 32611
}

\begin{abstract}
DOI: $10.2514 / 1 . A 32040$
A method is presented for costate estimation in nonlinear optimal control problems using multiple-interval collocation at Legendre-Gauss or Legendre-Gauss-Radau points. Transformations from the Lagrange multipliers of the nonlinear programming problem to the costate of the continuous-time optimal control problem are given. When the optimal costate is continuous, the transformed adjoint systems of the nonlinear programming problems are discrete representations of the continuous-time first-order optimality conditions. If, however, the optimal costate is discontinuous, then the transformed adjoint systems are not discrete representations of the continuous-time firstorder optimality conditions. In the case where the costate is discontinuous, the accuracy of the costate approximation depends on the locations of the mesh points. In particular, the accuracy of the costate approximation is found to be significantly higher when mesh points are located at discontinuities in the costate. Two numerical examples are studied and demonstrate the effectiveness of using the multiple-interval collocation approach for estimating costate in continuous-time nonlinear optimal control problems.
\end{abstract}

\section{Nomenclature}

C = path constraint function

D $=$ Gauss or Radau pseudospectral state differentiation matrix

$\mathbf{D}^{\dagger}=$ Gauss or Radau pseudospectral costate differentiation matrix

f $=$ state dynamics function

$g \quad=$ integrand of cost functional

$H=$ augmented Hamiltonian

$J \quad=$ continuous-time cost functional

$K=$ number of mesh intervals

$\mathcal{L}=$ Lagrangian of the nonlinear programming problem

$L(\tau)=$ Lagrange polynomial on time domain $\tau \in[-1,+1]$

$m \quad=$ dimension of continuous-time control

$n=$ dimension of continuous-time state

$N_{k}=$ number of collocation points in mesh interval $k$

$q=$ dimension of boundary condition function

$s=$ dimension of path constraint function

$t=$ time in interval $\left[t_{0}, t_{f}\right]$

$t_{f} \quad=$ final time

$t_{0} \quad=$ initial time

$\mathbf{u}(t)=$ control on time domain $t \in\left[t_{0}, t_{f}\right]$

$\mathbf{u}(\tau)=$ control on time domain $\tau \in[-1,+1]$

$\mathbf{U}_{j}=$ control approximation at time point $\tau_{j}$

$w_{j}=j$ th Legendre-Gauss or Legendre-Gauss-Radau quadrature weight

$\mathbf{X}(\tau)=$ state approximation on time domain $\tau \in[-1,+1]$

$\mathbf{x}(t)=$ state on time domain $t \in\left[t_{0}, t_{f}\right]$

Presented as Paper 2011-6571 at the 2011 Guidance, Navigation, and Control Conference, Portland, OR, 8-11 August 2011; received 1 February 2011; revision received 18 April 2011; accepted for publication 24 April 2011. Copyright $(\odot 2011$ by Anil Vithala Rao, Christopher Liu Darby, and Divya Garg. Published by the American Institute of Aeronautics and Astronautics, Inc., with permission. Copies of this paper may be made for personal or internal use, on condition that the copier pay the $\$ 10.00$ per-copy fee to the Copyright Clearance Center, Inc., 222 Rosewood Drive, Danvers, MA 01923; include the code 0022-4650/11 and \$10.00 in correspondence with the CCC.

*Ph.D. Student, Department of Mechanical and Aerospace Engineering; cdarby@ufl.edu.

${ }^{\dagger}$ Ph.D. Student, Department of Mechanical and Aerospace Engineering; divyagarg2002@ufl.edu.

II is noted that the first two authors on this paper share first authorship.

${ }^{\S}$ Assistant Professor, Department of Mechanical and Aerospace Engineering; anilvrao@ufl.edu (Corresponding Author).

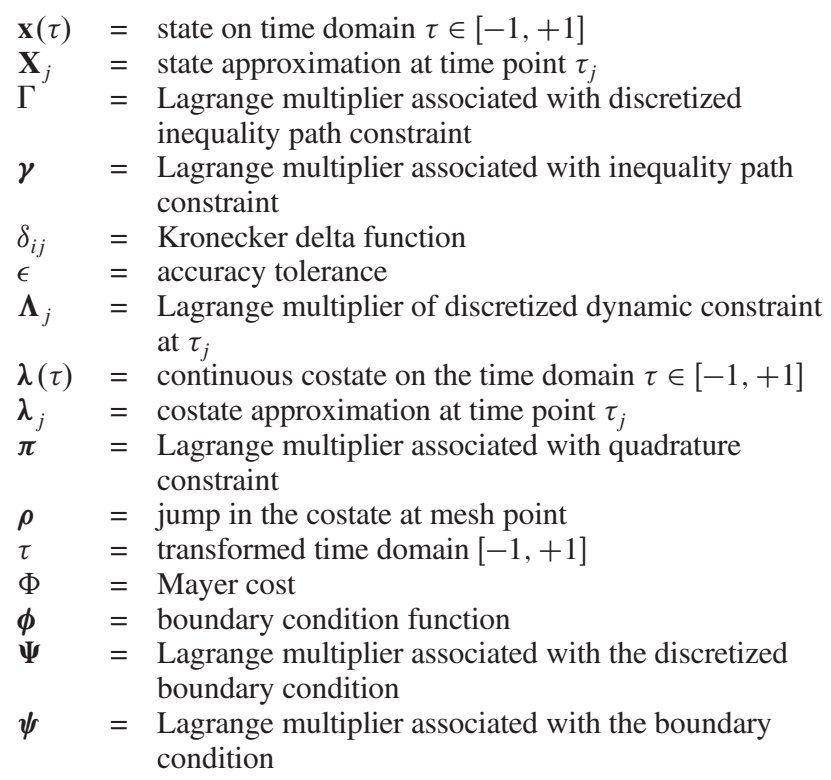

\section{Introduction}

N THE past two decades, direct collocation methods have become the preferred approach for solving optimal control problems. In a direct collocation method, the state and/or the control is approximated using trial (basis) functions and the continuous problem is transcribed to a finite-dimensional nonlinear programming problem (NLP). A particular class of direct collocation methods that has become popular in the last decade is that of pseudospectral methods [1-18]. In a pseudospectral method, the state is approximated using a basis of Lagrange polynomials and the differential-algebraic constraint equations are enforced at a finite set of collocation points. The three most commonly used sets of collocation points for pseudospectral methods are the Legendre-Gauss (LG) [1012,14,18], Legendre-Gauss-Radau (LGR) [13-15,17,18], and Legendre-Gauss-Lobatto (LGL) $[1,3,4,9,19]$ points. These points are the roots of the linear combinations of a Legendre polynomial and/or its derivatives and correspond to the three different types of Gaussian quadrature. All three sets of points are defined on the interval $[-1,1]$ but differ in how the endpoints are incorporated. LG points do not include the points -1 or +1 ; the LGR points include one of the endpoints, and the LGL points include both the endpoints. 
Pseudospectral methods have typically been employed in three different ways: $h$ methods, $p$ methods, and $h p$ methods. In an $h$ method, the optimal control problem is divided into many mesh intervals and a fixed low-degree polynomial is used in each mesh interval. Convergence in an $h$ method is then achieved by refining the mesh without changing the degree of the polynomial approximation in a mesh interval. In a $p$ method, a small number of approximating mesh intervals (often a single mesh interval) are used and convergence is achieved by increasing the degree of the polynomial approximation in each mesh interval. If the solution in each mesh interval is smooth, a $p$ method converges at an exponential rate. Finally, an $h p$ method is a combination of $h$ and $p$ methods, in that both the number of mesh intervals and the degree of the approximating polynomial in each mesh interval are allowed to change in order to achieve convergence to the solution of the optimal control problem. An advantage to using an $h$ method over a $p$ method is that an $h$ method NLP is more sparse than a $p$ method NLP. Recent research [17], however, has demonstrated that both accuracy and computational efficiency can be gained by using an $h p$ method over using either an $h$ method or a $p$ method.

Several different $h, p$, and $h p$ pseudospectral methods have been previously developed, with $p$ methods being the most studied of the

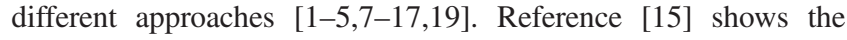
convergence rates for an $h$ LGR method. References $[1-5,7,7,8,8-$ 14,19] focus on $p$ methods using LG, LGR, and LGL collocation points. Reference [16] describes a $p$ biased $h p$ LG method with the key result being that a multitude of problems were accurately solved for state and control using fewer collocation points and greater sparsity as compared against a single-interval LG $p$ method. Reference [17] describes an $h$ biased LGR $h p$ method. The key result of [17] is that it is advantageous to use higher-degree collocation locally when it is known that the solution within a particular mesh interval is smooth. Moreover, the approach of [17] leads to a grid refinement algorithm where the computational sparsity of an $h$ method is retained while simultaneously improving the convergence rate in regions where the solution is smooth.

While the research of $[16,17]$ has demonstrated that $h p$ methods provide accurate approximations to the state and control, no methodology has been developed for costate estimation using a general multiple-interval formulation of a pseudospectral method. The motivation of this research is to analyze the effectiveness of $h p$ costate estimation methods using LG and LGR collocation points. To this end, the objective of this paper is to derive a costate estimation approach for continuous-time nonlinear optimal control problems using multiple-interval LG and LGR pseudospectral methods and to assess the accuracy of the approach. In a manner similar to that of $[16,17]$, the continuous-time optimal control problem is transcribed to an NLP using multiple-interval versions of the Gauss and Radau (that is, LG and LGR) pseudospectral methods [10-14]. The Karush-Kuhn-Tucker (KKT) conditions of the LG and L $\overline{\text { GR NLPs }}$ are then derived. Transformations of the NLP Lagrange multipliers to the costate of the continuous-time optimal control problem are then derived, leading to multiple-interval LG and LGR transformed adjoint systems [20]. Because the multiple-interval methods derived in this paper require continuity in the state at the mesh points, the formulation is significantly different from previously developed $p$ method costate estimation procedures such as those found in $[4,11,13,14]$. Specifically, for a continuous optimal costate it is found that the $\overline{\mathrm{LG}}$ and LGR transformed adjoint systems are discrete representations of the continuous-time first-order optimality conditions. When the optimal costate is discontinuous, however, the transformed adjoint systems are not discrete representations of the continuous-time first-order optimality conditions if a mesh point is at the location of discontinuity in the costate. In this latter case, it is found by example that the costate estimate is significantly more accurate when mesh points are located at the discontinuities than when mesh points are not placed at the discontinuities. Two examples are studied to analyze the accuracy of the costate estimation methods derived in this paper. In particular, the second example is a common type of problem that arises in aerospace engineering of minimumenergy control subject to a state inequality path constraint.
This paper is organized as follows. In Sec. II, we define our notation and conventions. In Sec. III, we state both the optimal control problem being approximated by our $h p$ approach and the corresponding first-order optimality conditions. In Sec. IV, we define the NLP that arises from the $h p$ LG and $h p$ LGR collocation methods used to discretize the continuous Bolza problem defined in Sec. III. In addition, we derive the first-order optimality conditions of these two NLPs and provide a transformation of the NLP Lagrange multipliers to the costate of the continuous-time optimal control problem. In Sec. $\mathrm{V}$, we study the accuracy of the costate estimation procedure on two examples. The first example has a smooth solution, while the second example has a discontinuous optimal costate. Finally, in Secs. VI and VII, we give a discussion of results and conclusions, respectively.

\section{Conventions and Notation}

For each method derived in this paper, $\left(\tau_{1}, \ldots, \tau_{N_{k}}\right) \in[-1,+1]$ denote the LG or LGR quadrature (collocation) points in interval $k$ [21]. It should be noted that the LG quadrature points lie on the interval $(-1,+1)$, while the LGR quadrature points lie on the interval $[-1,+1)$. To properly account for the boundary conditions, the LG method includes the noncollocated points $\tau_{0}=-1$ and $\tau_{N_{k}+1}=+1$, while the LGR method includes the noncollocated point $\tau_{N_{k}+1}=+1$. The manner in which these noncollocated points are used has been described in great detail in $[13,14,18]$. For either method, the state in mesh interval $k$ is approximated using a basis of Lagrange polynomials:

$$
L_{i}(\tau)=\prod_{\substack{l=Z \\ l \neq i}}^{N_{k}+Z} \frac{\tau-\tau_{l}^{(k)}}{\tau_{i}-\tau_{l}^{(k)}}, \quad Z \leq i \leq N_{k}
$$

where $Z=0$ for the LG method, and $Z=1$ for the LGR method. Next, all vector functions of time are denoted as row vectors; that is,

$$
\mathbf{y}(\tau)=\left[y_{1}(\tau), \cdots, y_{n}(\tau)\right] \in \mathbb{R}^{n}
$$

We define the approximation of the state, control, and the NLP Lagrange multipliers corresponding to the discrete approximation to the dynamics at $\tau=\tau_{i}$ as $\mathbf{X}_{i}, \mathbf{U}_{i}$, and $\boldsymbol{\Lambda}_{i}$, respectively. Moreover, in the LG method, $\mathbf{X}^{(k)}$ is an $\left(N_{k}+1\right) \times n$ matrix corresponding to the state at the initial point plus the LG points in mesh interval $k$, while in the LGR method, $\mathbf{X}^{(k)}$ is an $\left(N_{k}+1\right) \times n$ matrix corresponding to the state at the LGR points and the final point in mesh interval $k$. Similarly, $\mathbf{U}^{(k)}$ and $\boldsymbol{\Lambda}^{(k)}$ are $N_{k} \times n$ matrices that correspond to the control and NLP Lagrange multipliers, respectively, at either the LG or LGR points in mesh interval $k$. Finally, the notation $\mathbf{A}_{i: j}$ attached to any matrix $\mathbf{A}$ denotes rows $i$ through $j$ of the matrix $\mathbf{A}$, and $\mathbf{A}_{i}$ denotes row $i$ of the matrix $\mathbf{A}$.

In addition to the above vector and matrix conventions, the operation $\langle\mathbf{a}, \mathbf{b}\rangle$ is used to denote the standard inner product between the vectors $\mathbf{a} \in \mathbb{R}^{n}$ and $\mathbf{b} \in \mathbb{R}^{n}$. Furthermore, if $\mathbf{f}: \mathbb{R}^{n} \rightarrow \mathbb{R}^{m}$, then $\nabla \mathbf{f}$ is the $m$ by $n$ Jacobian matrix for which the $i$ th row is $\nabla \mathbf{f}_{i}$. Using this convention, the gradient of a scalar-valued function is a row vector. If $\phi: \mathbb{R}^{m \times n} \rightarrow \mathbb{R}$ and $\mathbf{X}$ is an $m$ by $n$ matrix, then $\nabla \phi$ denotes the $m$ by $n$ matrix for which the $(i, j)$ element is $(\nabla \phi(\mathbf{X}))_{i j}=\partial \phi(\mathbf{X}) / \partial X_{i j}$. Finally, the Kronecker delta function is defined by $\delta_{i i}=1$ and $\delta_{i j}=0$ if $i \neq j$.

\section{Bolza Optimal Control Problem}

Without loss of generality, consider the following fixed-time nonlinear optimal control problem in Bolza form. Minimize the cost functional

$$
J=\Phi\left(\mathbf{x}\left(t_{0}\right), \mathbf{x}\left(t_{f}\right)\right)+\int_{t_{0}}^{t_{f}} g(\mathbf{x}(t), \mathbf{u}(t)) \mathrm{d} t
$$




$$
\frac{\mathrm{d} \mathbf{x}}{\mathrm{d} t}=\mathbf{f}(\mathbf{x}(t), \mathbf{u}(t))
$$

the inequality path constraints

$$
\mathbf{C}(\mathbf{x}(t), \mathbf{u}(t)) \leq \mathbf{0}
$$

and the boundary conditions (i.e., the event constraints)

$$
\boldsymbol{\phi}\left(\mathbf{x}\left(t_{0}\right), \mathbf{x}\left(t_{f}\right)\right)=\mathbf{0}
$$

where $\quad \mathbf{x}(t) \in \mathbb{R}^{n}, \quad \mathbf{u}(t) \in \mathbb{R}^{m}, \quad \mathbf{C}: \mathbb{R}^{n} \times \mathbb{R}^{m} \rightarrow \mathbb{R}^{s}, \quad \boldsymbol{\phi}: \mathbb{R}^{n} \times$ $\mathbb{R}^{n} \rightarrow \mathbb{R}^{q}$, and $t_{0}$ and $t_{f}$ are fixed. Furthermore, we consider only problems where the functions $g, \mathbf{f}$, and $\mathbf{C}$ are not explicit functions of time, and the functions $\Phi$ and $\phi$ are not explicit functions of $t_{0}$ and $t_{f}$. It is noted, however, that the results shown in this paper are easily extendable to problems with a varying initial and/or final time and/or problems where time appears explicitly in Eqs. (2-5).

Suppose now that the continuous Bolza problem of Eqs. (2-5) is divided into $K$ mesh intervals $\left[t_{k-1}, t_{k}\right], k=1, \ldots, K$, where $\bar{t}_{0}<$ $t_{1}<t_{2}<\cdots<t_{K}=t_{f}$ are the mesh points. In each mesh interval, $t \in\left[t_{k-1}, t_{k}\right]$ is transformed to $\tau \in[-1,+1]$ via the affine transformation:

$$
\tau=\frac{2 t-\left(t_{k}+t_{k-1}\right)}{t_{k}-t_{k-1}}
$$

from which we obtain

$$
\frac{\mathrm{d} \tau}{\mathrm{d} t}=\frac{2}{t_{k}-t_{k-1}}, \quad 1 \leq k \leq K
$$

Next, let $\mathbf{x}^{(k)}(\tau)$ and $\mathbf{u}^{(k)}(\tau)$ be the state and control, respectively, in mesh interval $k$. Using Eq. (7), the Bolza optimal control problem in Eqs. (2-5) can be written as follows. First, the cost functional of Eq. (2) can be written as

$$
\begin{aligned}
J & =\Phi\left(\mathbf{x}^{(1)}(-1), \mathbf{x}^{(K)}(+1)\right) \\
& +\sum_{k=1}^{K} \frac{t_{k}-t_{k-1}}{2} \int_{-1}^{+1} g\left(\mathbf{x}^{(k)}(\tau), \mathbf{u}^{(k)}(\tau)\right) \mathrm{d} \tau
\end{aligned}
$$

Next, the dynamics of Eq. (3) and the path constraints of Eq. (4) are given in terms of $\tau$ in mesh interval $k \in[1, \ldots, K]$, respectively, as

$$
\begin{gathered}
\frac{\mathrm{d} \mathbf{x}^{(k)}(\tau)}{\mathrm{d} \tau} \equiv \dot{\mathbf{x}}^{(k)}(\tau)=\frac{t_{k}-t_{k-1}}{2} \mathbf{f}\left(\mathbf{x}^{(k)}(\tau), \mathbf{u}^{(k)}(\tau)\right) \\
\mathbf{C}\left(\mathbf{x}^{(k)}(\tau), \mathbf{u}^{(k)}(\tau)\right) \leq \mathbf{0}
\end{gathered}
$$

Furthermore, the boundary conditions of Eq. (ㅁ) are given as

$$
\boldsymbol{\phi}\left(\mathbf{x}^{(1)}(-1), \mathbf{x}^{(K)}(+1)\right)=\mathbf{0}
$$

Finally, the state is assumed to be continuous at an interior mesh point; that is,

$$
\mathbf{x}^{(k)}(+1)=\mathbf{x}^{(k+1)}(-1), \quad 1 \leq k \leq K-1
$$

We now state the first-order optimality conditions from the calculus of variations for the multiple-interval continuous-time Bolza optimal control problem as given in Eqs. (י-12). First, the augmented Hamiltonian in interval $k$ is defined as

$$
H^{(k)}\left(\mathbf{x}^{(k)}, \lambda^{(k)}, \mathbf{u}^{(k)}, \boldsymbol{\gamma}^{(k)}\right)=g^{(k)}+\left\langle\lambda^{(k)}, \mathbf{f}^{(k)}\right\rangle-\left\langle\boldsymbol{\gamma}^{(k)}, \mathbf{C}^{(k)}\right\rangle
$$

where $\lambda^{(k)}(\tau) \in \mathbb{R}^{n}$ and $\boldsymbol{\gamma}^{(k)}(\tau) \in \mathbb{R}^{s}$. Applying the continuous-time first-order optimality conditions from the calculus of variations $[\underline{22}, \underline{23}]$, we obtain

$$
\nabla_{\mathbf{u}} H^{(k)}=\nabla_{\mathbf{u}}\left(g^{(k)}+\left\langle\lambda^{(k)}, \mathbf{f}^{(k)}\right\rangle-\left\langle\boldsymbol{\gamma}^{(k)}, \mathbf{C}^{(k)}\right\rangle\right)=\mathbf{0}
$$

$$
\begin{aligned}
-\frac{t_{k}-t_{k-1}}{2} \nabla_{\mathbf{x}} H^{(k)} & =-\frac{t_{k}-t_{k-1}}{2} \nabla_{\mathbf{x}}\left(g^{(k)}+\left\langle\lambda^{(k)}, \mathbf{f}^{(k)}\right\rangle\right. \\
& \left.-\left\langle\boldsymbol{\gamma}^{(k)}, \mathbf{C}^{(k)}\right\rangle\right)=\dot{\lambda}^{(k)}(\tau)
\end{aligned}
$$

$$
\lambda^{(1)}(-1)=-\nabla_{\mathbf{x}^{(1)}(-1)}(\boldsymbol{\Phi}-\langle\boldsymbol{\psi}, \boldsymbol{\phi}\rangle)
$$

$$
\lambda^{(K)}(1)=\nabla_{\mathbf{x}^{(K)}(+1)}(\boldsymbol{\Phi}-\langle\boldsymbol{\psi}, \boldsymbol{\phi}\rangle)
$$

$$
\lambda^{(k)}(1)=\lambda^{(k+1)}(-1)+\rho^{(k)}, \quad 1 \leq k \leq K-1
$$

where $\psi \in \mathbb{R}^{q}$, and $\rho^{(k)} \in \mathbb{R}^{n}$ is the jump (discontinuity) in the costate at the mesh points due to active path constraints and interior point constraints. Then, from the complementary slackness condition, the Lagrange multiplier $\boldsymbol{\gamma}^{(k)}$ takes the values

$$
\begin{array}{lll}
\gamma_{j}^{(k)}(\tau)=0 & \text { when } C_{j}^{(k)}(\tau)<0, & 1 \leq j \leq s \\
\gamma_{j}^{(k)}(\tau)<0 & \text { when } C_{j}^{(k)}(\tau)=0, & 1 \leq j \leq s
\end{array}
$$

\section{Legendre-Gauss and Legendre-Gauss-Radau Discretizations of Bolza Problem}

In this section, we consider the discretization of the continuous Bolza optimal control problem of Sec. III using multiple-interval versions of the previously developed Gauss (LG) and Radau (LGR) pseudospectral methods [10-14]. First, the optimal control problem is transcribed to a finite-dimensional NLP for each discretization method. Next, the KKT conditions of the LG and LGR NLPs, along with the corresponding transformed adjoint systems [20], are derived for the $h p$ LG and $h p$ LGR methods. We show that if the costate is continuous, the transformed adjoint systems for both the LG and LGR $h p$ methods are discrete approximations of the continuous-time first-order optimality conditions. If, however, the costate is discontinuous, we show that the LG and LGR transformed adjoint systems are not discrete approximations of the continuous-time firstorder optimality conditions.

\section{A. Legendre-Gauss Discretization}

In the Gauss (LG) pseudospectral method, the state is approximated in each mesh interval $k$ as

$$
\mathbf{X}^{(k)}(\tau) \approx \mathbf{X}^{(k)}(\tau)=\sum_{j=0}^{N_{k}} \mathbf{X}_{j}^{(k)} L_{j}^{(k)}(\tau)
$$

Differentiating $\mathbf{X}^{(k)}(\tau)$ in Eq. $(\underline{21})$ with respect to $\tau$, we obtain

$$
\frac{\mathrm{d} \mathbf{X}^{(k)}(\tau)}{\mathrm{d} \tau} \equiv \dot{\mathbf{X}}^{(k)}(\tau)=\sum_{j=0}^{N_{k}} \mathbf{X}_{j}^{(k)} \dot{L}_{j}^{(k)}(\tau)
$$

Collocating the right-hand side of the state dynamics of Eq. (9) with the derivative of the state approximation in Eq. (22) at the $\bar{N}_{k} \mathrm{LG}$ points, we have

$$
\sum_{j=0}^{N_{k}} \mathbf{X}_{j}^{(k)} D_{i j}^{(k)}-\frac{t_{k}-t_{k-1}}{2} \mathbf{f}_{i}^{(k)}=\mathbf{0}, \quad 1 \leq i \leq N_{k}
$$

where $\mathbf{f}_{i}^{(k)}=\mathbf{f}\left(\mathbf{X}_{i}^{(k)}, \mathbf{U}_{i}^{(k)}\right)$ and

$$
D_{i j}^{(k)}=\dot{L}_{j}^{(k)}\left(\tau_{i}^{(k)}\right), \quad 1 \leq i \leq N_{k}, \quad 0 \leq j \leq N_{k}
$$

is the $N_{k} \times\left(N_{k}+1\right)$ Gauss pseudospectral differentiation matrix $[\underline{7}, \underline{10}-12]$ in the $k$ th mesh interval. Next, the cost functional of Eq. (ㅇ) is approximated using a multiple-interval LG quadrature as 


$$
J \approx \Phi\left(\mathbf{X}_{0}^{(1)}, \mathbf{X}_{N_{K}+1}^{(K)}\right)+\sum_{k=1}^{K} \sum_{i=1}^{N_{k}} \frac{t_{k}-t_{k-1}}{2} w_{i}^{(k)} g_{i}^{(k)}
$$

where $g_{i}^{(k)}=g\left(\mathbf{X}_{i}^{(k)}, \mathbf{U}_{i}^{(k)}\right)$. The path constraints of Eq. (10) are enforced at the $N_{k}$ LG points in mesh interval $k$ as

$$
\mathbf{C}_{i} \equiv \mathbf{C}\left(\mathbf{X}_{i}^{(k)}, \mathbf{U}_{i}^{(k)}\right) \leq \mathbf{0}, \quad 1 \leq i \leq N_{k}
$$

Finally, the boundary conditions are approximated as

$$
\boldsymbol{\phi}\left(\mathbf{X}_{0}^{(1)}, \mathbf{X}_{N_{K}+1}^{(K)}\right)=\mathbf{0}
$$

In addition, an NLP variable corresponding to the state at the terminal point of each mesh interval $k, \mathbf{X}^{(k)}(+1)=\mathbf{X}_{N_{k}+1}^{(k)}$, is included by adding the following LG quadrature approximation to the state at $\tau=+1$ :

$$
\mathbf{X}_{N_{k}+1}^{(k)}=\mathbf{X}_{0}^{(k)}+\frac{t_{k}-t_{k-1}}{2} \sum_{i=1}^{N_{k}} w_{i}^{(k)} \mathbf{f}_{i}^{(k)}, \quad 1 \leq k \leq K
$$

It is noted that state continuity at the mesh points $k \in[1, \ldots, K-1]$ is enforced via the constraint

$$
\mathbf{X}_{N_{k}+1}^{(k)}=\mathbf{X}_{0}^{(k+1)}
$$

When implementing the Gauss pseudospectral method, a single variable is used for the value of the state at the end of mesh interval $k$ and the start of mesh interval $k+1$; that is, $\mathbf{X}_{N_{k}+1}^{(k)} \equiv \mathbf{X}_{0}^{(k+1)}$, $1 \leq k \leq K-1$. Hence, redundant variables defining the state at the interior mesh points are eliminated in the discretization and Eq. (28) can be written as

$$
\begin{aligned}
& \mathbf{X}_{0}^{(k+1)}=\mathbf{X}_{0}^{(k)}+\frac{t_{k}-t_{k-1}}{2} \sum_{i=1}^{N_{k}} w_{i}^{(k)} \mathbf{f}_{i}^{(k)}, \quad 1 \leq k \leq K-1 \\
& \mathbf{X}_{N_{K}+1}^{(K)}=\mathbf{X}_{0}^{(K)}+\frac{t_{K}-t_{K-1}}{2} \sum_{i=1}^{N_{K}} w_{i}^{(K)} \mathbf{f}_{i}^{(K)}
\end{aligned}
$$

The NLP that arises from the Gauss pseudospectral approximation is to minimize the cost function of Eq. (25) subject to the algebraic constraints of Eqs. (23), (26), (27), and (푸).

\section{B. Karush-Kuhn-Tucker Conditions for Legendre-Gauss \\ Discretization}

The KKT conditions of the NLP of Sec. IV.A are now derived. The Lagrangian of the NLP is given as

$$
\begin{aligned}
\mathcal{L} & =\Phi\left(\mathbf{X}_{0}^{(1)}, \mathbf{X}_{N_{K}+1}^{(K)}\right)-\left\langle\Psi, \boldsymbol{\phi}\left(\mathbf{X}_{0}^{(1)}, \mathbf{X}_{N_{K}+1}^{(K)}\right\rangle\right. \\
& +\sum_{k=1}^{K} \sum_{j=1}^{N_{k}}\left(\frac{t_{k}-t_{k-1}}{2} w_{j}^{(k)} g_{j}^{(k)}-\left\langle\Gamma_{j}^{(k)}, \mathbf{C}_{j}^{(k)}\right\rangle\right. \\
& \left.-\left\langle\boldsymbol{\Lambda}_{j}^{(k)}, \mathbf{D}_{j, 1: N_{k}}^{(k)} \mathbf{X}_{1: N_{k}}^{(k)}+D_{j, 0}^{(k)} \mathbf{X}_{0}^{(k)}-\frac{t_{k}-t_{k-1}}{2} \mathbf{f}_{j}^{(k)}\right\rangle\right) \\
& -\sum_{k=1}^{K-1}\left\langle\boldsymbol{\pi}^{(k)}, \mathbf{X}_{0}^{(k+1)}-\mathbf{X}_{0}^{(k)}-\frac{t_{k}-t_{k-1}}{2} \sum_{j=1}^{N_{k}} w_{j}^{(k)} \mathbf{f}_{j}^{(k)}\right\rangle \\
& -\left\langle\boldsymbol{\pi}^{(K)}, \mathbf{X}_{N_{k}+1}^{(K)}-\mathbf{X}_{0}^{(K)}-\frac{t_{K}-t_{K-1}}{2} \sum_{j=1}^{N_{K}} w_{j}^{(K)} \mathbf{f}_{j}^{(K)}\right\rangle
\end{aligned}
$$

where $\boldsymbol{\Lambda}^{(k)}, \Gamma^{(k)}, \boldsymbol{\Psi}$, and $\boldsymbol{\pi}^{(k)}$ are the Lagrange multipliers associated, respectively, with the discretized dynamic constraints of Eq. (23), the discretized path constraints of Eq. (26), the discretized event constraints of Eq. (27), and the quadrature constraints of Eq. (30) in mesh interval $k$. The KKT conditions of the NLP are obtained by differentiating $\mathcal{L}$ with respect to $\mathbf{X}_{j}^{(k)}, 0 \leq j \leq N_{k}+1$, and $\mathbf{U}_{j}^{(k)}$, $1 \leq j \leq N_{k}$, in each interval $k$ and setting these derivatives equal to zero. The KKT conditions are given as

$$
\begin{gathered}
\mathbf{0}=\nabla_{\mathbf{U}}\left(w_{j}^{(k)} g_{j}^{(k)}+\left\langle\boldsymbol{\Lambda}_{j}^{(k)}+w_{j}^{(k)} \boldsymbol{\pi}^{(k)}, \mathbf{f}_{j}^{(k)}\right\rangle\right. \\
\left.-\frac{2}{t_{k}-t_{k-1}}\left\langle\Gamma_{j}^{(k)}, \mathbf{C}_{j}^{(k)}\right\rangle\right), \quad 1 \leq k \leq K, \quad 1 \leq j \leq N_{k} \\
\mathbf{D}_{j}^{(k)} \boldsymbol{\Lambda}^{(k)}=\frac{t_{k}-t_{k-1}}{2} \nabla_{\mathbf{X}}\left(w_{j}^{(k)} g_{j}^{(k)}+\left\langle\boldsymbol{\Lambda}_{j}^{(k)}+w_{j}^{(k)} \boldsymbol{\pi}^{(k)}, \mathbf{f}_{j}^{(k)}\right\rangle\right. \\
\left.-\frac{2}{t_{k}-t_{k-1}}\left\langle\Gamma_{j}^{(k)}, \mathbf{C}_{j}^{(k)}\right\rangle\right), \quad 1 \leq k \leq K, \quad 1 \leq j \leq N_{k} \\
\mathbf{D}_{0}^{(1)^{\top}} \boldsymbol{\Lambda}^{(1)}-\boldsymbol{\pi}^{(1)}=\nabla_{\mathbf{X}_{0}^{(1)}}(\Phi-\langle\boldsymbol{\Psi}, \boldsymbol{\phi}\rangle) \\
\mathbf{D}_{0}^{(k)^{\top}} \boldsymbol{\Lambda}^{(k)}-\boldsymbol{\pi}^{(k)}=-\boldsymbol{\pi}^{(k-1)}, \quad 2 \leq k \leq K \\
\boldsymbol{\pi}^{(K)}=\nabla_{\mathbf{X}_{N_{K}+1}^{(k)}}(\Phi-\langle\boldsymbol{\Psi}, \boldsymbol{\phi}\rangle)
\end{gathered}
$$

where $\mathbf{D}_{j}^{\top}$ is the $j$ th row of $\mathbf{D}^{\top}$. Next, consider the following change of variables from continuous to discrete time:

$$
\begin{aligned}
& \psi=\Psi \\
& \boldsymbol{\gamma}_{j}^{(k)}=\frac{\Gamma_{j}^{(k)}}{w_{j}^{(k)}} \frac{2}{t_{k}-t_{k-1}}, \quad 1 \leq k \leq K, \quad 1 \leq j \leq N_{k} \\
& \lambda_{j}^{(k)}=\frac{\boldsymbol{\Lambda}^{(k)}}{w_{j}^{(k)}}+\boldsymbol{\pi}^{(k)}, \quad 1 \leq k \leq K, \quad 1 \leq j \leq N_{k}
\end{aligned}
$$

Furthermore, define the matrix $\mathbf{D}^{\dagger(k)}$ as

$$
\begin{gathered}
D_{i j}^{\dagger(k)}=-\frac{w_{j}^{(k)}}{w_{i}^{(k)}} D_{j i}^{(k)}, \quad 1 \leq k \leq K, \quad 1 \leq i, j \leq N_{k} \\
D_{i, N_{k}+1}^{\dagger(k)}=-\sum_{j=1}^{N_{k}} D_{i j}^{\dagger(k)}, \quad 1 \leq i \leq N_{k}
\end{gathered}
$$

From Eq. (36), the costate approximation at $t=t_{f}$ is obtained as

$$
\lambda_{N_{K}+1}^{(K)}=\pi^{(K)}
$$

Next, let

$$
\lambda_{0}^{(1)}=-\mathbf{D}_{0}^{(1)^{\top}} \boldsymbol{\Lambda}^{(1)}+\boldsymbol{\pi}^{(1)}
$$

be the costate approximation at $t=t_{0}$. Substituting the change of variables given in Eqs. (37) and (1-43) into Eqs. (32) and (36), the LG transformed adjoint system is given as

$$
\begin{gathered}
\mathbf{0}=\nabla_{\mathbf{U}} H\left(\mathbf{X}_{j}^{(k)}, \mathbf{U}_{j}^{(k)}, \boldsymbol{\gamma}_{j}^{(k)}, \lambda_{j}^{(k)}\right), \quad 1 \leq k \leq K, \quad 1 \leq j \leq N_{k} \\
\left(\mathbf{D}_{j, 1: N_{k}}^{\dagger(k)} \lambda^{(k)}+D_{j, N_{k}+1}^{\dagger(k)} \pi^{(k)}\right)=-\frac{t_{k}-t_{k-1}}{2} \\
\quad \times \nabla_{\mathbf{X}} H\left(\mathbf{X}_{j}^{(k)}, \mathbf{U}_{j}^{(k)}, \boldsymbol{\gamma}_{j}^{(k)}, \lambda_{j}^{(k)}\right) \\
1 \leq k \leq K, \quad 1 \leq j \leq N_{k} \\
\lambda_{0}^{(1)}=-\nabla_{\mathbf{X}_{0}^{(1)}}(\Phi-\langle\boldsymbol{\psi}, \boldsymbol{\phi}\rangle) \\
\lambda_{N_{K}+1}^{(K)}=\nabla_{\mathbf{X}_{N_{K}+1}^{(K)}}(\Phi-\langle\boldsymbol{\psi}, \boldsymbol{\phi}\rangle)
\end{gathered}
$$




$$
\begin{gathered}
\boldsymbol{\pi}^{(k-1)}=\boldsymbol{\pi}^{(k)}+\frac{t_{k}-t_{k-1}}{2} \sum_{j=1}^{N_{k}} w_{j}^{(k)} \nabla_{\mathbf{X}} H\left(\mathbf{X}_{j}^{(k)}, \mathbf{U}_{j}^{(k)}, \boldsymbol{\gamma}_{j}^{(k)}, \lambda_{j}^{(k)}\right) \\
2 \leq k \leq K
\end{gathered}
$$

where it is noted that Eq. (48) is formed by comparing Eqs. (33) and (35) together with the identity [14]

$$
D_{i, 0}^{(k)}=-\sum_{j=1}^{N_{k}} D_{i j}^{(k)}, \quad 1 \leq i \leq N_{k}
$$

Next, setting $k=K$ in Eq. (48) and noting that $\lambda_{N_{K}+1}^{(K)}=\pi^{(K)}$, we obtain

$$
\lambda_{0}^{(K)}=\boldsymbol{\pi}^{(K-1)}
$$

Suppose now that the costate is continuous at the mesh points $k=1, \ldots, K-1$. Then, the right-hand side of Eq. (48) is an approximation for the costate at the beginning of mesh intervals $k=1, \ldots, K-1$; that is,

$$
\lambda_{0}^{(k+1)}=\lambda_{N_{k}+1}^{(k)}=\pi^{(k)}, \quad(k=1, \ldots, K-1)
$$

It is noted that the transformed adjoint system is a discrete representation of the continuous-time first-order necessary conditions if the costate is continuous at every mesh point.

Suppose now that the costate is discontinuous at a particular mesh point $M \in[1, \ldots, K-1]$. Then,

$$
\boldsymbol{\pi}^{(M)}=\lambda_{0}^{(M+1)}=\lambda_{N_{M}+1}^{(M)}-\boldsymbol{\rho}^{(M)}
$$

where $\rho^{(M)}$ is the difference between $\lambda_{N_{M}+1}^{(M)}$ and $\lambda_{0}^{(M+1)}$. Therefore, if the costate is discontinuous at mesh point $M, \pi^{(M)} \neq \lambda_{N_{M}+1}^{(M)}$ in Eq. (45). Hence, the left-hand side of Eq. (45) is not a discrete approximation to the continuous-time costate dynamics. As a result, the transformed adjoint system given in Eqs. (44-48) is not a discrete representation of the first-order optimality conditions given in Eqs. (14-18).

\section{Legendre-Gauss-Radau Discretization}

In the Radau (LGR) pseudospectral method, the state is approximated in each mesh interval $k$ as

$$
\mathbf{X}^{(k)}(\tau) \approx \mathbf{X}^{(k)}(\tau)=\sum_{j=1}^{N_{k}+1} \mathbf{X}_{j}^{(k)} L_{j}^{(k)}(\tau)
$$

Differentiating $\mathbf{X}^{(k)}(\tau)$ in Eq. $(\underline{53})$ with respect to $\tau$, we obtain

$$
\frac{\mathrm{d} \mathbf{X}^{(k)}(\tau)}{\mathrm{d} \tau} \equiv \dot{\mathbf{X}}^{(k)}(\tau)=\sum_{j=1}^{N_{k}+1} \mathbf{X}_{j}^{(k)} \dot{L}_{j}^{(k)}(\tau)
$$

Collocating the state dynamics of Eq. (9) with the derivative of the state approximation in Eq. (54) at the $N_{k}$ LGR points, we have

$$
\sum_{j=1}^{N_{k}+1} \mathbf{X}_{j}^{(k)} D_{i j}^{(k)}-\frac{t_{k}-t_{k-1}}{2} \mathbf{f}_{i}^{(k)}=\mathbf{0}, \quad 1 \leq i \leq N_{k}
$$

where

$$
D_{i j}^{(k)}=\dot{L}_{j}^{(k)}\left(\tau_{i}^{(k)}\right), \quad 1 \leq i \leq N_{k}, \quad 1 \leq j \leq N_{k}+1
$$

is the $N_{k} \times\left(N_{k}+1\right)$ Radau pseudospectral differentiation matrix [13] in mesh interval $k$. The cost functional of Eq. ( ) is then approximated using a multiple-interval LGR quadrature as

$$
J \approx \Phi\left(\mathbf{X}_{1}^{(1)}, \mathbf{X}_{N_{K}+1}^{(K)}\right)+\sum_{k=1}^{K} \sum_{i=1}^{N_{k}} \frac{t_{k}-t_{k-1}}{2} w_{i}^{(k)} g_{i}^{(k)}
$$

Furthermore, the path constraints of Eq. (4) are enforced at the $N_{k}$ LGR points in mesh interval $k$ as

$$
\mathbf{C}_{i}^{(k)} \equiv \mathbf{C}\left(\mathbf{X}_{i}^{(k)}, \mathbf{U}_{i}^{(k)}\right) \leq \mathbf{0}, \quad 1 \leq i \leq N_{k}
$$

Finally, the boundary conditions are approximated as

$$
\boldsymbol{\phi}\left(\mathbf{X}_{1}^{(1)}, \mathbf{X}_{N_{K}+1}^{(K)}\right)=\mathbf{0}
$$

Continuity in the state at the mesh points $k \in[1, \ldots, K-1]$ is imposed via the equality constraint

$$
\mathbf{X}_{N_{k}+1}^{(k)}=\mathbf{X}_{1}^{(k+1)}
$$

The NLP that arises from the Radau pseudospectral approximation is to minimize the cost function of Eq. (57) subject to the algebraic constraints of Eqs. (55) and (58-60). As with the multiple-interval form of the Gauss pseudospectral method, the same NLP variable is used for both $\mathbf{X}_{N_{k}+1}^{(k)}$ and $\mathbf{X}_{1}^{(k+1)}$, thus eliminating the need for Eq. (60) and removing any redundant variables in the discretization. The constraints of Eq. (55) are then written as

$$
\begin{aligned}
& \mathbf{D}_{j, 1: N_{k}}^{(k)} \mathbf{X}_{1: N_{k}}^{(k)}+D_{j, N_{k}+1}^{(k)} \mathbf{X}_{1}^{(k+1)}-\frac{t_{k}-t_{k-1}}{2} \mathbf{f}_{j}^{(k)}=\mathbf{0} \\
& \quad 1 \leq k \leq K-1 \\
& \mathbf{D}_{j, 1: N_{K}}^{(K)} \mathbf{X}_{1: N_{K}}^{(K)}+D_{j, N_{K}+1}^{(K)} \mathbf{X}_{N_{K}+1}^{(K)}-\frac{t_{K}-t_{K-1}}{2} \mathbf{f}_{j}^{(K)}=\mathbf{0}
\end{aligned}
$$

\section{Karush-Kuhn-Tucker Conditions for Legendre-Gauss-Radau Discretization}

The KKT conditions of the NLP of Sec. IV.C are now derived. The Lagrangian of the LGR NLP is given as

$$
\begin{aligned}
\mathcal{L} & =\Phi\left(\mathbf{X}_{1}^{(1)}, \mathbf{X}_{N_{K}+1}^{(K)}\right)-\left\langle\boldsymbol{\Psi}, \boldsymbol{\phi}\left(\mathbf{X}_{1}^{(1)}, \mathbf{X}_{N_{K}+1}^{(K)}\right\rangle\right. \\
& +\sum_{k=1}^{K} \sum_{j=1}^{N_{k}} \frac{t_{k}-t_{k-1}}{2} w_{j}^{(k)} g_{j}^{(k)}-\left\langle\Gamma_{j}^{(k)}, \mathbf{C}_{j}^{(k)}\right\rangle \\
& -\sum_{k=1}^{K-1} \sum_{j=1}^{N_{k}}\left\langle\boldsymbol{\Lambda}_{j}^{(k)}, \mathbf{D}_{j, 1: N_{k}}^{(k)} \mathbf{X}_{1: N_{k}}^{(k)}+D_{j, N_{k}+1}^{(k)} \mathbf{X}_{1}^{(k+1)}\right. \\
& \left.-\frac{t_{k}-t_{k-1}}{2} \mathbf{f}_{j}^{(k)}\right\rangle-\sum_{j=1}^{N_{K}}\left\langle\boldsymbol{\Lambda}_{j}^{(K)}, \mathbf{D}_{j, 1: N_{K}}^{(K)} \mathbf{X}_{1: N_{K}}^{(K)}\right. \\
& \left.+D_{j, N_{K}+1}^{(K)} \mathbf{X}_{N_{K}+1}^{(K)}-\frac{t_{K}-t_{K-1}}{2} \mathbf{f}_{j}^{(K)}\right\rangle
\end{aligned}
$$

where $\boldsymbol{\Lambda}^{(k)}, \Gamma^{(K)}, \boldsymbol{\Psi}$, and $\pi^{(k)}$ are the Lagrange multipliers associated, respectively, with the discretized dynamic constraints of Eq. (55), the discretized path constraints of Eq. (58), and the discretized event constraints of Eq. (59) in mesh interval $k$. The KKT conditions of the multiple-interval L $\overline{\mathrm{GR}}$ discretization are then given as

$$
\begin{gathered}
\mathbf{0}=\nabla_{\mathbf{U}}\left(w_{j}^{(k)} g_{j}^{(k)}+\left\langle\boldsymbol{\Lambda}_{j}^{(k)}, \mathbf{f}_{j}^{(k)}\right\rangle-\frac{2}{t_{k}-t_{k-1}}\left\langle\Gamma_{j}^{(k)}, \mathbf{C}_{j}^{(k)}\right\rangle\right) \\
1 \leq k \leq K, \quad 1 \leq j \leq N_{k} \\
\mathbf{D}_{j}^{(1)^{\top}} \boldsymbol{\Lambda}^{(1)}=\frac{t_{1}-t_{0}}{2} \nabla_{\mathbf{X}}\left(w_{j}^{(1)} g_{j}^{(1)}+\left\langle\boldsymbol{\Lambda}_{j}^{(1)}, \mathbf{f}_{j}^{(1)}\right\rangle\right. \\
\left.-\frac{2}{t_{1}-t_{0}}\left\langle\Gamma_{j}^{(1)}, \mathbf{C}_{j}^{(1)}\right\rangle\right)-\delta_{1 j}\left(-\nabla_{\mathbf{X}_{1}^{(1)}} \Phi+\nabla_{\mathbf{X}_{1}^{(1)}}\langle\boldsymbol{\Psi}, \boldsymbol{\phi}\rangle\right) \\
1 \leq j \leq N_{1}
\end{gathered}
$$




$$
\begin{gathered}
\mathbf{D}_{j}^{(k)^{\top}} \boldsymbol{\Lambda}^{(k)}=\frac{t_{k}-t_{k-1}}{2} \nabla_{\mathbf{X}}\left(w_{j}^{(k)} g_{j}^{(k)}+\left\langle\boldsymbol{\Lambda}_{j}^{(k)}, \mathbf{f}_{j}^{(k)}\right\rangle\right. \\
\left.-\frac{2}{t_{k}-t_{k-1}}\left\langle\Gamma_{j}^{(k)}, \mathbf{C}_{j}^{(k)}\right\rangle\right)-\delta_{1 j} \mathbf{D}_{N_{k-1}+1}^{(k-1)^{\top}} \boldsymbol{\Lambda}^{(k-1)} \\
2 \leq k \leq K, \quad 1 \leq j \leq N_{k}
\end{gathered}
$$

$$
\mathbf{D}_{N_{K}+1}^{(K)^{\top}} \boldsymbol{\Lambda}^{(K)}=\nabla_{\mathbf{X}_{N_{K}+1}^{(K)}}(\Phi-\langle\Psi, \boldsymbol{\phi}\rangle)
$$

Next, consider the following change of variables from continuous to discrete time:

$$
\begin{gathered}
\boldsymbol{\psi}=\boldsymbol{\Psi} \\
\boldsymbol{\gamma}_{j}^{(k)}=\frac{2}{t_{k}-t_{k-1}} \frac{\Gamma_{j}^{(k)}}{w_{j}^{(k)}}, \quad 1 \leq k \leq K, \quad 1 \leq j \leq N_{k} \\
\lambda_{j}^{(k)}=\frac{\boldsymbol{\Lambda}_{j}^{(k)}}{w_{k}^{(k)}}, \quad 1 \leq k \leq K, \quad 1 \leq j \leq N_{k} \\
\boldsymbol{\pi}^{(k)}=\mathbf{D}_{N_{k}+1}^{(k)^{\top}} \boldsymbol{\Lambda}^{(k)}, \quad 1 \leq k \leq K
\end{gathered}
$$

In addition, let the matrix $\mathbf{D}^{\dagger(k)}$ be defined as

$$
\begin{gathered}
D_{11}^{\dagger(k)}=-D_{11}^{(k)}-\frac{1}{w_{1}^{(k)}} \\
D_{i j}^{\dagger(k)}=-\frac{w_{j}^{(k)}}{w_{i}^{(k)}} D_{j i}^{(k)} \quad \text { otherwise }
\end{gathered}
$$

Substituting Eqs. (67-71) and (12) into Eqs. (63-66), the transformed adjoint system is given as

$$
\mathbf{0}=\nabla_{\mathbf{U}} H\left(\mathbf{X}_{j}^{(k)}, \mathbf{U}_{j}^{(k)}, \lambda_{j}^{(k)}, \boldsymbol{\gamma}_{j}^{(k)}\right), \quad 1 \leq k \leq K, \quad 1 \leq j \leq N_{k}
$$

$$
\begin{gathered}
\mathbf{D}_{j}^{\dagger(1)} \boldsymbol{\lambda}^{(1)}=-\frac{t_{1}-t_{0}}{2} \nabla_{\mathbf{X}} H\left(\mathbf{X}_{j}^{(1)}, \mathbf{U}_{j}^{(1)}, \lambda_{j}^{(1)}, \boldsymbol{\gamma}_{j}^{(1)}\right) \\
+\frac{\delta_{1 j}}{w_{1}^{(1)}}\left(-\nabla_{\mathbf{X}_{1}^{(1)}} \Phi+\nabla_{\mathbf{X}_{1}^{(1)}}\langle\boldsymbol{\psi}, \boldsymbol{\phi}\rangle-\lambda_{1}^{(1)}\right) \\
\mathbf{D}_{j}^{\dagger(k)} \lambda^{(k)}=-\frac{t_{k}-t_{k-1}}{2} \nabla_{\mathbf{X}} H\left(\mathbf{X}_{j}^{(k)}, \mathbf{U}_{j}^{(k)}, \lambda_{j}^{(k)}, \boldsymbol{\gamma}_{j}^{(k)}\right) \\
+\frac{\delta_{1 j}}{w_{1}^{(k)}}\left(\boldsymbol{\pi}^{(k-1)}-\lambda_{1}^{(k)}\right), \quad 2 \leq k \leq K, \quad 1 \leq j \leq N_{k}
\end{gathered}
$$

$$
\pi^{(K)}=\nabla_{\mathbf{X}_{N_{K}+1}^{(K)}}(\boldsymbol{\Phi}-\langle\boldsymbol{\psi}, \boldsymbol{\phi}\rangle)
$$

Analogous to [13], we expand Eq. (70) using Eqs. (64) and (도) to obtain the following relationships:

$$
\begin{aligned}
& -\nabla_{\mathbf{X}_{1}^{(1)}} \Phi+\nabla_{\mathbf{X}_{1}^{(1)}}\langle\boldsymbol{\psi}, \boldsymbol{\phi}\rangle=\boldsymbol{\pi}^{(1)} \\
& +\frac{t_{1}-t_{0}}{2} \sum_{j=1}^{N_{1}} w_{j}^{(1)} \nabla_{\mathbf{x}} H\left(\mathbf{X}_{j}^{(1)}, \mathbf{U}_{j}^{(1)}, \lambda_{j}^{(1)}, \boldsymbol{\gamma}_{j}^{(1)}\right)
\end{aligned}
$$

$$
\begin{gathered}
\boldsymbol{\pi}^{(k-1)}=\boldsymbol{\pi}^{(k)}+\frac{t_{k}-t_{k-1}}{2} \sum_{j=1}^{N_{k}} w_{j}^{(k)} \nabla_{\mathbf{X}} H\left(\mathbf{X}_{j}^{(k)}, \mathbf{U}_{j}^{(k)}, \lambda_{j}^{(k)}, \boldsymbol{\gamma}_{j}^{(k)}\right) \\
2 \leq k \leq K
\end{gathered}
$$

Next, the costate approximation at $t=t_{f}$ is given as

$$
\lambda_{N_{K}+1}^{(K)}=\nabla_{\mathbf{x}_{N_{K}+1}^{(K)}}(\Phi-\langle\boldsymbol{\psi}, \boldsymbol{\phi}\rangle)
$$

from which we obtain

$$
\lambda_{N_{K}+1}^{(K)}=\pi^{(K)}
$$

The right-hand side of Eq. (78) for $k=K$ is then an approximation to $\lambda_{1}^{(K)}$; therefore, $\lambda_{1}^{(K)}=\pi^{(K-1)}$. Consequently, the final term in Eq. (75) disappears for $k=K$. Furthermore, if the costate is continuous at the mesh point $k=K-1$, we obtain

$$
\lambda_{N_{K-1}+1}^{(K-1)}=\lambda_{1}^{(K)}=\pi^{(K-1)}
$$

Furthermore, for $k=K-1$, the right-hand side of Eq. (78) becomes an approximation to $\lambda_{1}^{(K-1)}$. Thus, we have

$$
\lambda_{1}^{(K-1)}=\boldsymbol{\pi}^{(K-2)}
$$

If the costate is continuous at every mesh point, it then follows that

$$
\lambda_{N_{k}+1}^{(k)}=\lambda_{1}^{(k+1)}=\pi^{(k)}, \quad 1 \leq k \leq K-1
$$

As a result, the left-hand side of Eq. (77) is then an approximation to the costate at $t_{0}$. Therefore, for the case when the costate is continuous at every mesh point, the final term in Eqs. (74) and (75) disappears and the transformed adjoint system is a discrete representation of continuous-time first-order optimality conditions.

Suppose now that the costate is discontinuous at a particular mesh point $M \in[1, \ldots, K-1]$. Then,

$$
\boldsymbol{\pi}^{(M)}=\lambda_{1}^{(M+1)}=\lambda_{N_{M}+1}^{(M)}-\rho^{(M)}
$$

where $\rho^{(M)}$ is the difference between $\lambda_{N_{M}+1}^{(M)}$ and $\lambda_{1}^{(M+1)}$. Therefore, if the costate is discontinuous at mesh point $M, \pi^{(M-1)} \neq \lambda_{1}^{(M)}$ in Eq. (75). As a result, the transformed adjoint system given in Eqs. (73-76) is not a discrete representation of the first-order optimality conditions given in Eqs. (14-18).

\section{Examples}

The costate estimation methods derived in Sec. IV are now applied to two optimal control problems that have been studied extensively. In the first example, the optimal costate is smooth, while in the second example, the optimal costate is discontinuous. The purpose of the examples is to assess the accuracy of the multiple-interval LG and LGR costate estimation procedures as functions of the number of collocation points and the degree of the polynomial approximation and compare them against single-interval $p$ methods. A performance analysis on the methods is not given with respect to CPU time, number of collocation points, or NLP density. See [16,17] for performance analyses of multiple-interval LG and LGR methods.

The examples were solved using the open-source program General Pseudospectral Optimal Control Software [7] with the NLP solver SNOPT [24]. All computations were performed using a $2.5 \mathrm{GHz}$ Core 2 Duo Macbook Pro running Mac OS-X 10.5.8 with MATLAB $\mathrm{R} 2009 \mathrm{~b}$. The $p$ method discretizations were solved using a fixed number of intervals with a variable number of collocation points in each interval. For the first example and the first part of the second example, a single interval was used. In the second part of the second example, three intervals were used, and these three intervals were connected at the locations of the costate discontinuity. The $h$ method 
discretizations were solved using a fixed low-degree approximation in each interval using a variable number of intervals. The $h p$ method discretizations were solved using a variable number of collocation points per interval and a variable number of intervals. Throughout this section, the terminology $h-x$ denotes an $h$ method of degree $x$ in every mesh interval, while $h p-x$ denotes an $h p$ method with a minimum polynomial degree of $x$ in each interval.

\section{A. Example 1}

Consider the following optimal control problem taken from [13]. Minimize the cost functional

$$
J=\frac{1}{2} \int_{0}^{t_{f}}\left(y+u^{2}\right) \mathrm{d} t
$$

subject to the dynamic constraint

$$
\dot{y}=2 y+2 u \sqrt{y}
$$

and the boundary conditions

$$
y(0)=2, \quad y\left(t_{f}\right)=1
$$

where $t_{f}=5$. The optimal state and costate for this example are $y^{*}(t)=\left(x^{*}(t)\right)^{2}$ and $\lambda_{y}^{*}(t)=\lambda_{x}^{*}(t) /\left(2 x^{*}(t)\right)$, respectively, where $x^{*}(t)$ and $\lambda_{x}^{*}(t)$ are given as

$$
\left[\begin{array}{l}
x^{*}(t) \\
\lambda_{x}^{*}(t)
\end{array}\right]=\exp (\mathbf{A} t)\left[\begin{array}{c}
x_{0} \\
\lambda_{x 0}
\end{array}\right], \quad \mathbf{A}=\left[\begin{array}{cc}
1 & -1 \\
-1 & -1
\end{array}\right]
$$

and

$$
\begin{gathered}
x_{0}=\sqrt{2}, \quad x_{f}=1 \\
\mathbf{B}=\left[\begin{array}{ll}
B_{11} & B_{12} \\
B_{21} & B_{22}
\end{array}\right]=\exp \left(\mathbf{A} t_{f}\right) \\
\lambda_{x 0}=\frac{x_{f}-B_{11} x_{0}}{B_{12}}
\end{gathered}
$$

In this example we analyze the costate errors using the LG and LGR $p, h-2, h-3$, and $h-4$ methods. It is noted that the $h-x$ methods are constructed with a uniformly distributed grid of mesh points. Because this example has a smooth solution, it is expected that a $p$ method will converge the fastest. Furthermore, because the optimal costate is continuous, it is expected that the multiple-interval LG and LGR methods (i.e., $h$ ) will provide accurate approximations to the optimal costate because the transformed adjoint systems defined in Sec. IV are discrete representations of the continuous-time first-order optimality conditions.

Figures $1 \mathrm{a}$ and $1 \mathrm{~b}$ show the base 10 logarithm of the maximum absolute costate error as a function of the number of collocation points for LG and LGR $p$ and $h-x$ methods, respectively. Because the solution to this problem is smooth [that is, $\mathbf{x}^{*}(t), \mathbf{u}^{*}(t)$, and $\lambda^{*}(t)$ are all smooth], the $p$ method converges the fastest using either discretization scheme. Next, it is seen in Figs. 1a and $1 \mathrm{~b}$ that the convergence rate using the $h-x$ methods is much slower than the convergence rate using the $p$ methods. For the $h-x$ methods, the rate of convergence increases with the degree of approximating polynomial. A closer examination of Figs. $1 \mathrm{a}$ and $1 \mathrm{~b}$ also reveals that the costate estimate using an LG $h-x$ method converges faster than the corresponding costate estimate using an LGR $h-x$ method.

\section{B. Example 2}

Consider the following optimal control minimum-energy optimal control problem with an inequality state constraint taken from [23]. Minimize the cost functional

$$
J=\frac{1}{2} \int_{0}^{1} a^{2} \mathrm{~d} t
$$

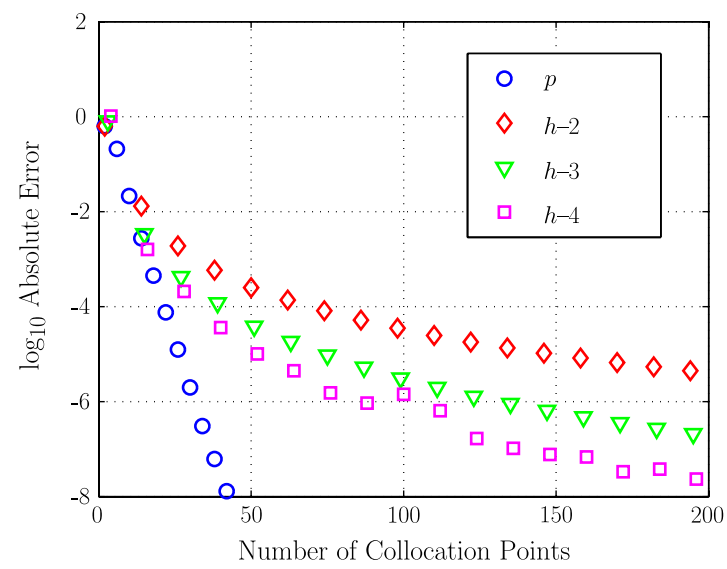

a)

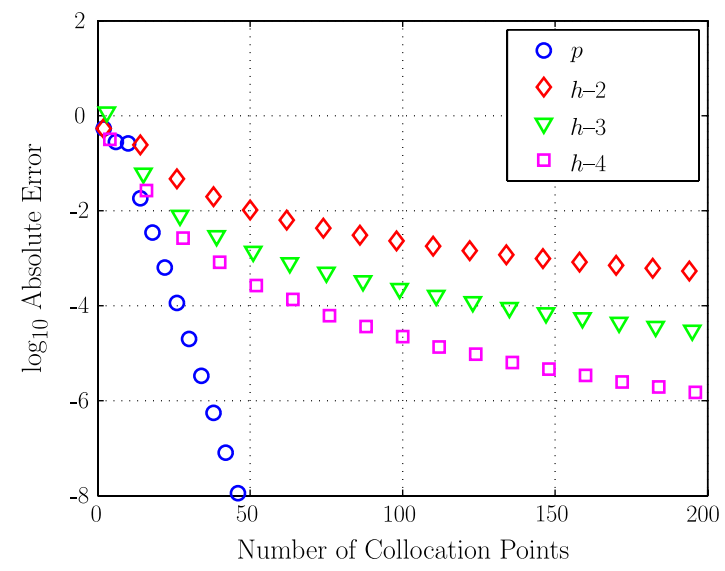

b)

Fig. 1 Absolute costate erors for a) LG and b) LGR points for example 1.

subject to the dynamic constraints

$$
\begin{aligned}
& \dot{x}=v \\
& \dot{v}=a
\end{aligned}
$$

the boundary conditions

$$
\begin{gathered}
x(0)=x(1)=0 \\
v(0)=-v(1)=1
\end{gathered}
$$

and the constraint $x(t) \leq \ell$. The optimal costate for this example is

$$
\begin{gathered}
\lambda_{x}^{*}= \begin{cases}\frac{2}{9 \ell^{2}}, & 0 \leq t \leq 3 \ell, \\
0, & 3 \ell \leq t \leq 1-3 \ell, \\
-\frac{2}{9 \ell^{2}}, & 1-3 \ell \leq t \leq 1\end{cases} \\
\lambda_{v}^{*}= \begin{cases}\frac{2}{3 \ell}\left(1-\frac{t}{3 \ell}\right) & 0 \leq t \leq 3 \ell, \\
0 & 3 \ell \leq t \leq 1-3 \ell, \\
\frac{2}{3 \ell}\left(1-\frac{1-t}{3 \ell}\right) & 1-3 \ell \leq t \leq 1\end{cases}
\end{gathered}
$$

Unlike the optimal solution to example 1, where the optimal solution was smooth, it is seen in Eqs. (97) and (98) that $\lambda_{x}^{*}(t)$ is discontinuous while $\lambda_{v}^{*}(t)$ is continuous but not smooth. In this example, we study the numerical approximations obtained for $\ell=1 / 12$. Furthermore, we consider discretizations where no mesh points are at the locations of the discontinuity in $\lambda_{x}^{*}(t)$ and discretizations where mesh points are located at the discontinuity in $\lambda_{x}^{*}(t)$. 


\section{Case 1: No Mesh Points at Costate Discontinuity}

In this case, we analyze LG and LGR $p$, uniformly spaced $h-2$, and $h p-3$ methods. In general, mesh points will not be located exactly at a costate discontinuity. Figures $2 \mathrm{a}$ and $3 \mathrm{~b}$ show the base 10 logarithm of the maximum absolute error at the collocation points for the costate as a function of the number of collocation points using LG and LGR $p$ methods and uniformly distributed $h-2$ methods, respectively. First, it is seen that essentially no difference is seen in the convergence rates of the LG and LGR $p$ and $h-2$ methods. Next, while $\lambda_{v}$ converges (albeit slowly), the first component of the costate does not converge when a mesh point is not located at the discontinuity.

Suppose now that we attempt to improve the convergence of the costate by applying the $h p-3$ method of [17] to this example, where it is noted that the $h p$-adaptive method of [17] is designed to capture discontinuities in the control and potential nonsmoothness in the state. Table 1 shows the maximum costate errors for various accuracy tolerances $\epsilon$, while Figs. $\underline{4 a}$ and $\underline{4 b}$ show the costate approximation and the location of the mesh points on the final grid for $\epsilon=10^{-4}$. While the $h p$-adaptive method places mesh points near the costate discontinuity, it is seen that the approximation still leads to inaccuracies at the point of the costate discontinuity [in particular, see the value of $\lambda_{x}(t)$ at $t \approx 0.25$ in Fig. 4a]. Thus, even though the $h p$ adaptive method refines the mesh near the discontinuity in $\lambda_{x}$, the errors in $\lambda_{x}$ are still large near the discontinuities because no mesh points are located at the discontinuity itself. Finally, it is noted for $\epsilon=10^{-6}$ that the errors in the state and control are $\mathcal{O}\left(10^{-6}\right)$ and $\mathcal{O}\left(10^{-3}\right)$, respectively. This last fact demonstrates that placing the mesh points near but not exactly at the point of the costate discontinuity significantly decreases the error in the state and control but has a significantly lesser effect on the accuracy of the costate. In

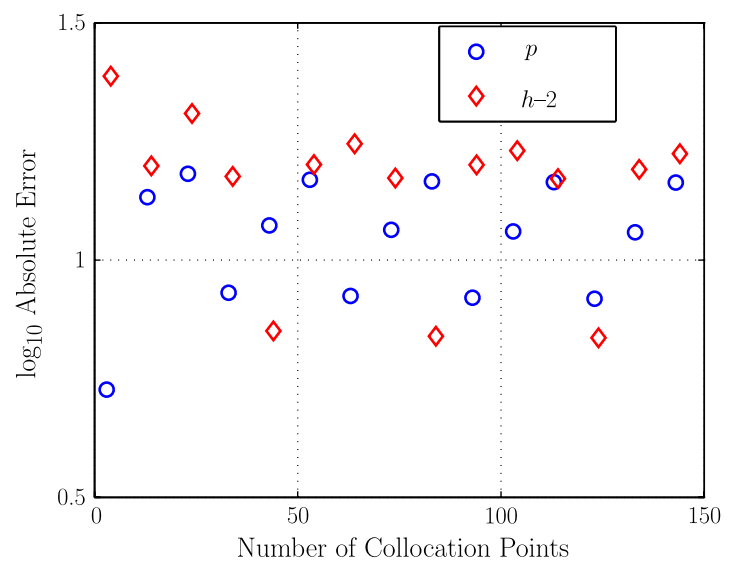

a)

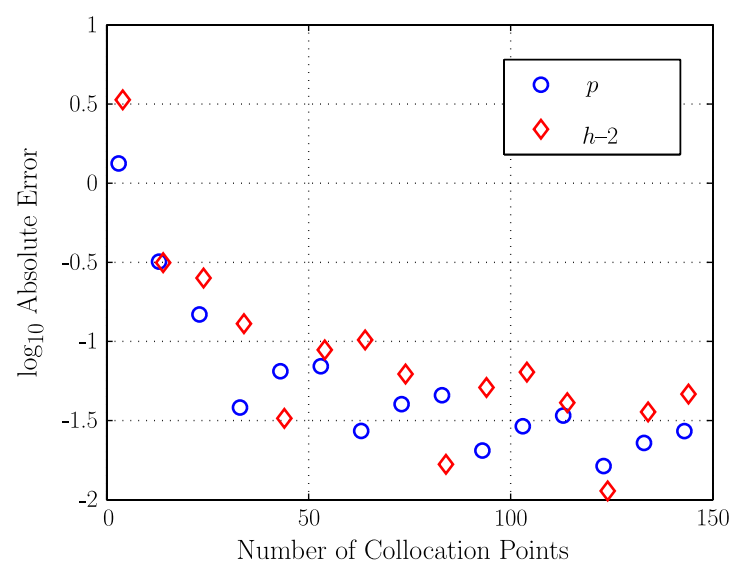

b)

Fig. 2 Maximum absolute error in a) $\lambda_{x}^{*}(t)$ and b) $\lambda_{v}^{*}(t)$ vs number of collocation points using $h-2$ and $p$ LG methods for example 2 when mesh points are not located at the costate discontinuity.

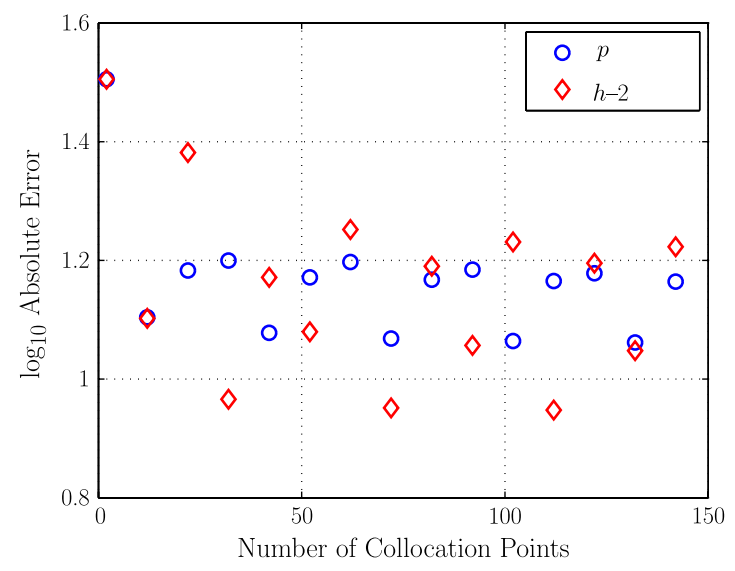

a)

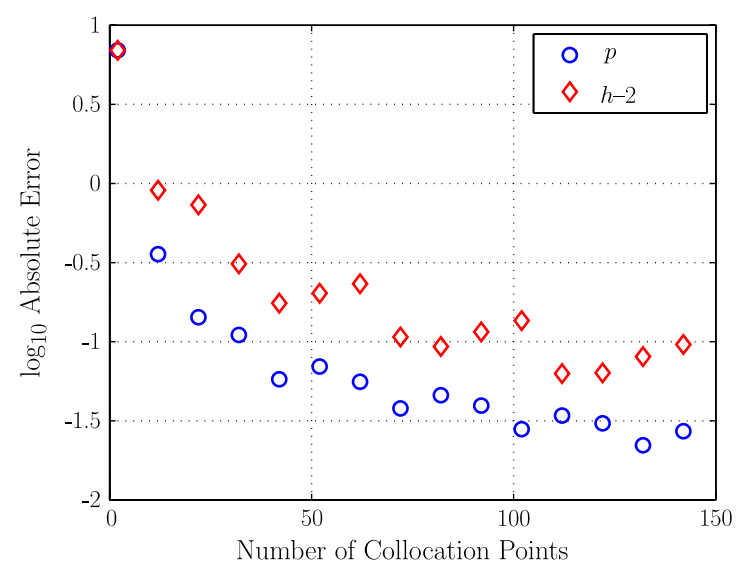

b)

Fig. 3 Maximum absolute error in a) $\lambda_{x}^{*}(t)$ and b) $\lambda_{v}^{*}(t)$ vs number of collocation points using $h-2$ and $p$ LGR methods for example 2 when mesh points are not located at the costate discontinuity.

the next section, we show the results when a mesh point is located at the location of the costate discontinuity.

\section{Case 2: Location of Costate Discontinuity Known}

Suppose now that we use the fact that we know the locations of the discontinuities in $\lambda_{x}^{*}(t)$ to aid in improving the accuracy of the costate. Specifically, let us apply the $h-3$ and $p$ methods to this problem by dividing the time interval into three segments: $T_{1}=[0,3 \ell], T_{2}=[3 \ell, 1-3 \ell]$, and $T_{3}=[1-3 \ell, 1]$. In the $h-3$ method, $T_{1}$ and $T_{3}$ are divided into uniformly spaced intervals while three collocation points are used in $T_{2}$. In the $p$ method, a single mesh interval is used in each segment $T_{1}, T_{2}$, and $T_{3}$.

Figures 5 and 6 show the maximum costate errors using the LG, LGR $h-\overline{3}$, and $\bar{p}$ methods as a function of the number of mesh intervals (using the $h-3$ method) and as a function of the polynomial degree (for the $p$ method) when a mesh point is located at each of the costate discontinuities. It is seen that the accuracy in the costate is improved dramatically when mesh points coincide with the discontinuities. More interesting, the LG method produces errors that are near machine precision using a small number of mesh intervals

Table 1 Summary of maximum absolute costate errors for example 2 using an $h p-3$ method for various accuracy tolerances $\epsilon$

\begin{tabular}{lccc}
\hline \hline$\epsilon$ & $\lambda_{x}$ & $\lambda_{v}$ & $\begin{array}{c}\text { Number of Collocation } \\
\text { Points }\end{array}$ \\
\hline $10^{-2}$ & $1.39 \times 10^{1}$ & $2.02 \times 10^{-1}$ & 30 \\
$10^{-4}$ & $2.32 \times 10^{1}$ & $2.99 \times 10^{-2}$ & 58 \\
$10^{-6}$ & $1.30 \times 10^{1}$ & $2.32 \times 10^{-3}$ & 87 \\
\hline \hline
\end{tabular}




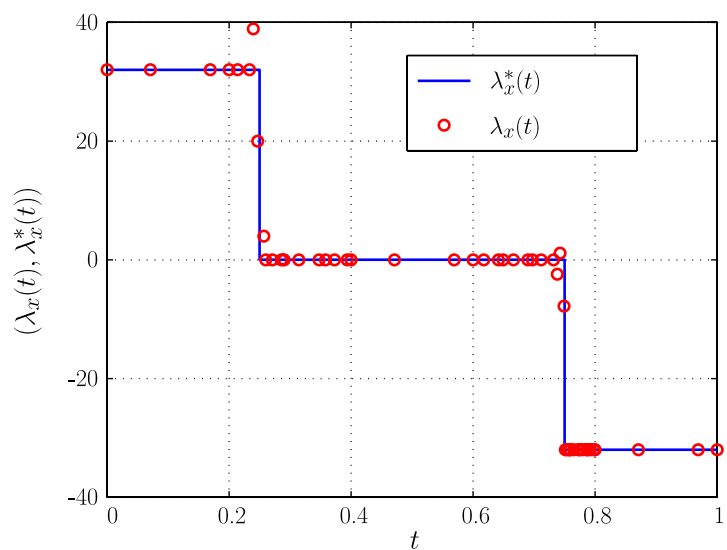

a)

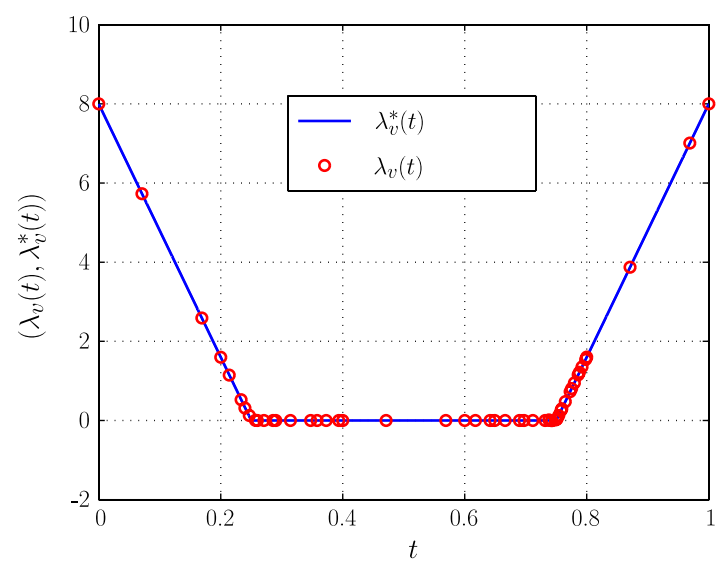

b)

Fig. 4 Costate approximation using $h p-3$ method with $\epsilon=10^{-4}$ for example 2 alongside optimal solution.

(in the case of the $h-3$ method) or using a small polynomial degree (in the case of the $p$ method). Moreover, as the number of approximating intervals or the degree of approximation is increased, it is interesting to see the accuracy of the LG method decreases. For the LGR method, as the number of approximating intervals or degree of approximation is increased, the LGR method does not significantly increase or decrease in accuracy. Rather, the LGR accuracy lies between $\mathcal{O}\left(10^{-4}\right)$ and $\mathcal{O}\left(10^{-12}\right)$. Next, comparing Figs. 5a and $5 \mathrm{~b}$, it is seen that the errors in the two components of the costate are nearly identical using an $h-3$ method. Thus, even though only $\lambda_{x}^{*}(t)$ is discontinuous while $\lambda_{v}^{*}(t)$ is continuous, the size of the errors in the two components of the costate are the same because the transformed adjoint system is not a discrete representation of the costate dynamics. Furthermore, Figs. $6 \mathrm{a}$ and $6 \mathrm{~b}$ show that by using $\mathrm{LG}$ and LGR $p$ methods, the approximation of $\overline{\lambda_{v}^{*}}(t)$ is slightly more accurate than the approximation of $\lambda_{x}^{*}(t)$.

\section{Discussion}

The two examples studied in Sec. $\underline{\mathrm{V}}$ demonstrate some of the key characteristics of the LG and LGR $h \bar{p}$ schemes. In example 1, where the solution is smooth, the LG and LGR costate approximations converge using $h, p$, and $h p$ methods. These results are consistent with the fact that the costate is continuous, thereby making the LG and LGR transformed adjoint system discrete representations of the continuous-time first-order optimality conditions. Furthermore, and consistent with the fact that the solution to example 1 is smooth, a $p$ method converges the most rapidly of all of the different discretization methods.

Next, the results obtained for example 2, where the optimal costate is discontinuous, are quite different from those obtained in example 1 . When mesh points are not located at the discontinuities,

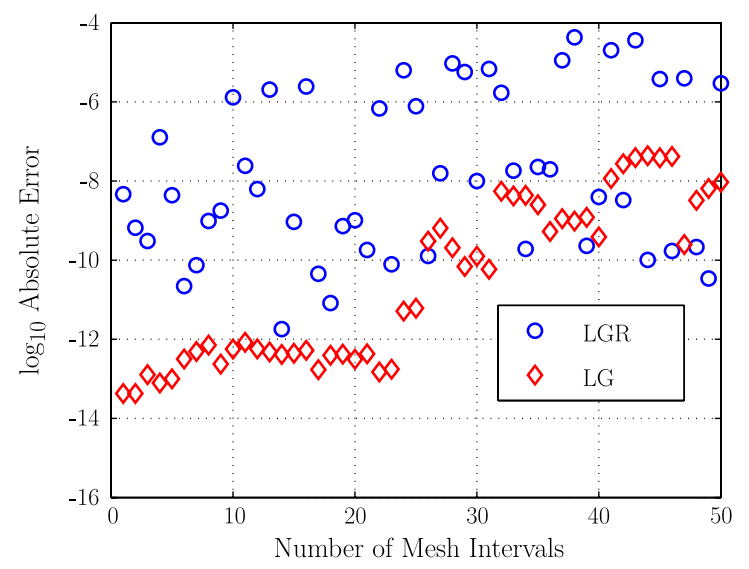

a)

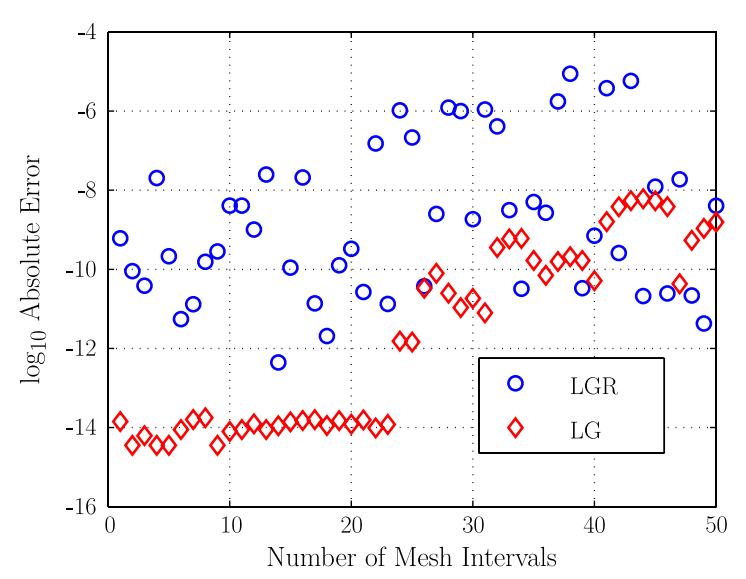

b)

Fig. 5 Absolute costate errors for a) $\lambda_{x}^{*}(t)$ and b) $\lambda_{v}^{*}(t)$ vs number of mesh intervals in segments $T_{1}$ and $T_{3}$ using LG and LGR $h-3$ methods when mesh points are located at the costate discontinuity.

the discontinuous components of the costate do not converge for any of the methods. Furthermore, even for the components of the costate that are continuous, the convergence is extremely slow. To improve the costate approximation in example 2 , mesh points are located at the costate discontinuities. While improving the placement of the mesh points still does not result in the transformed adjoint systems being discrete representations of the continuous-time first-order optimality conditions, the accuracy of the LG and LGR costate approximations is much higher with proper mesh point placement than it was when no mesh points were located at the costate discontinuities. Finally, even though in example 2 the second component of the optimal costate was continuous, the errors in this second costate component were similar to those of the first (discontinuous) costate component.

In general, it is expected that the costate approximation methods derived in this paper will lead to accurate approximations for problems for which the optimal costate are continuous. In the case where the optimal costate is discontinuous, however, the accuracy is expected to depend greatly on the placement of the mesh points. In particular, if mesh points are not placed at the discontinuities in the costate, it is expected that the costate errors will be large even if the corresponding errors in the state and control are significantly smaller.

\section{Conclusions}

Methods have been presented for costate estimation using multiple-interval collocation at LG and LGR points. A mapping from the Lagrange multipliers of the multiple-interval discretized optimal control problem to the costate of the continuous-time optimal control problem has been derived for each method. The requirement 


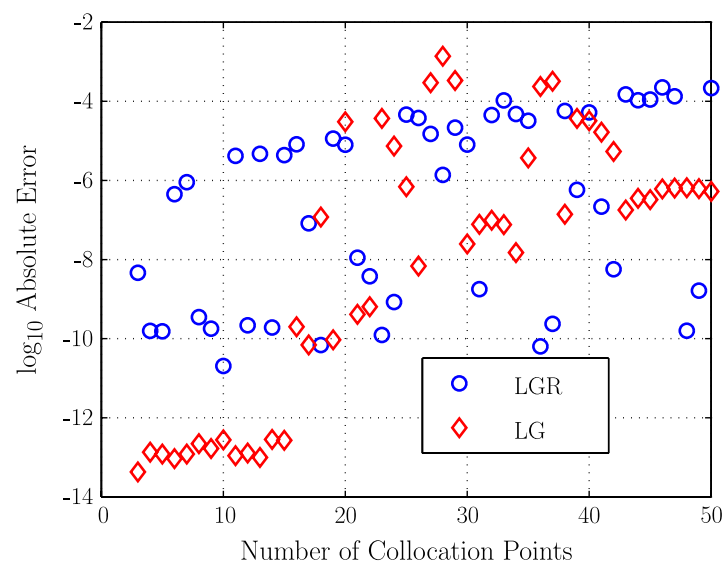

a)

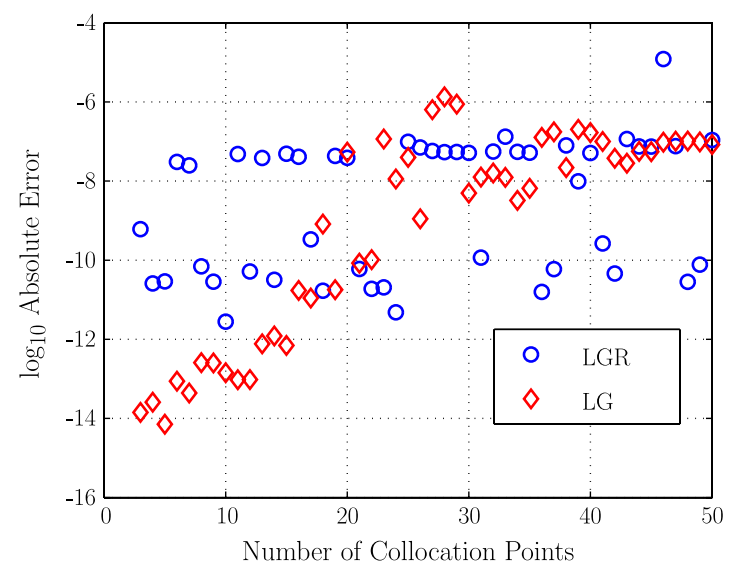

b)

Fig. 6 Absolute costate errors for a) $\lambda_{x}^{*}(t)$ and b) $\lambda_{v}^{*}(t)$ vs number of collocation points in segments $T_{1}$ and $T_{3}$ using LG and LGR $p$ methods when mesh points are located at the costate discontinuity.

to enforce continuity in the state at the mesh points leads to a significantly different transformation of the Lagrange multipliers of the pseudospectral NLP than is obtained using a global pseudospectral method. If the costate is continuous, the transformed adjoint system is a discrete representation of the continuous-time first-order optimality conditions. If, however, the costate is discontinuous, the transformed adjoint system is not an exact discrete representation of the continuous-time first-order optimality conditions. It was found that, even though, in the case of a discontinuous costate, the transformed adjoint system was not a discrete representation of the continuous-time first-order optimality conditions, the accuracy of the approximation could be improved if mesh points were placed at the locations of the discontinuities.

\section{Acknowledgments}

The authors gratefully acknowledge partial support for this research from the NASA Florida Space Grant Consortium under grant NNG05GK00H and from the U.S. Office of Naval Research under grant N00014-11-1-0068.

\section{References}

[1] Elnagar, G., Kazemi, M., and Razzaghi, M., "The Pseudospectral Legendre Method for Discretizing Optimal Control Problems," IEEE Transactions on Automatic Control, Vol. 40, No. 10, 1995, pp. $1793-$ 1796.

doi:10.1109/9.467672

[2] Elnagar, G., and Kazemi, M., "Pseudospectral Chebyshev Optimal Control of Constrained Nonlinear Dynamical Systems," Computational Optimization and Applications, Vol. 11, No. 2, 1998, pp. 195-217.
doi:10.1023/A:1018694111831

[3] Fahroo, F., and Ross, I. M., "A Spectral Patching Method for Direct Trajectory Optimization," Journal of the Astronautical Sciences, Vol. 48, Nos. 2-3, April-Sept. 2000, pp. 269-286.

[4] Fahroo, F., and Ross, I. M., "Costate Estimation by a Legendre Pseudospectral Method," Journal of Guidance, Control, and Dynamics, Vol. 24, No. 2, 2001, pp. 270-277. doi: $10.2514 / 2.4709$

[5] Ross, I. M., and Fahroo, F., "Pseudospectral Knotting Methods for Solving Optimal Control Problems," Journal of Guidance, Control, and Dynamics, Vol. 27, No. 3, 2004, pp. 397-405. doi: $10.2514 / 1.3426$

[6] Gong, Q., Ross, I. M., Kang, W., and Fahroo, F., "Connections Between the Covector Mapping Theorem and Convergence of Pseudospectral Methods," Computational Optimization and Applications, Vol. 41, No. 3, Dec. 2008, pp. 307-335. doi:10.1007/s10589-007-9102-4

[7] Rao, A. V., Benson, D. A., Darby, C. L., Francolin, C., Patterson, M. A., Sanders, I., and Huntington, G. T., "Algorithm 902: GPOPS, A Matlab Software for Solving Multiple-Phase Optimal Control Problems Using the Gauss Pseudospectral Method," ACM Transactions on Mathematical Software, Vol. 37, No. 2, April-June 2010, pp. 22:122:39. doi:10.1145/1731022.1731032

[8] Williams, P., "Jacobi Pseudospectral Method for Solving Optimal Control Problems," Journal of Guidance, Control, and Dynamics, Vol. 27, No. 2, 2004, pp. 293-297. doi:10.2514/1.4063

[9] Williams, P., "Application of Pseudospectral Methods for Receding Horizon Control," Journal of Guidance, Control, and Dynamics, Vol. 27, No. 2, 2004, pp. 310-314. doi: $10.2514 / 1.5118$

[10] Benson, D. A., "A Gauss Pseudospectral Transcription for Optimal Control," Ph.D. Thesis, Department of Aeronautics and Astronautics, Massachusetts Inst. of Technology, Cambridge, MA, 2004.

[11] Benson, D. A., Huntington, G. T., Thorvaldsen, T. P., and Rao, A. V., "Direct Trajectory Optimization and Costate Estimation via an Orthogonal Collocation Method," Journal of Guidance, Control, and Dynamics, Vol. 29, No. 6, Nov.-Dec. 2006, pp. 1435-1440. doi:10.2514/1.20478

[12] Huntington, G. T., "Advancement and Analysis of a Gauss Pseudospectral Transcription for Optimal Control," Ph.D. Thesis, Massachusetts Inst. of Technology, Cambridge, MA, 2007.

[13] Garg, D., Patterson, M. A., Darby, C. L., Francolin, C., Huntington, G. T., Hager, W. W., and Rao, A. V., "Direct Trajectory Optimization and Costate Estimation of Finite-Horizon and Infinite-Horizon Optimal Control Problems Using a Radau Pseudospectral Method," Computational Optimization and Applications, Vol. 49, No. 2, June 2011, pp. 335-358.

doi:10.1007/s10589-009-9291-0

[14] Garg, D., Patterson, M. A., Hager, W. W., Rao, A. V., Benson, D. A., and Huntington, G. T., "A Unified Framework for the Numerical Solution of Optimal Control Problems Using Pseudospectral Methods," Automatica, Vol. 46, No. 11, Nov. 2010, pp. 1843-1851. doi:10.1016/j.automatica.2010.06.048

[15] Kameswaran, S., and Biegler, L. T., "Convergence Rates for Direct Transcription of Optimal Control Problems Using Collocation at Radau Points," Computational Optimization and Applications, Vol. 41, No. 1, Sept. 2008, pp. 81-126. doi:10.1007/s10589-007-9098-9

[16] Darby, C. L., Hager, W. W., and Rao, A. V., "An hp-Adaptive Pseudospectral Method for Solving Optimal Control Problems," Optimal Control Applications and Methods, Vol. 32, No. 4, JulyAug. 2011, pp. 476-502. doi:10.1002/oca.957

[17] Darby, C. L., Hager, W. W., and Rao, A. V., "Direct Trajectory Optimization Using a Variable Low-Order Adaptive Pseudospectral Method," Journal of Spacecraft and Rockets, Vol. 48, No. 3, MayJune 2011, pp. 433-445. doi: $10.2514 / 1.52136$

[18] Garg, D., Hager, W. W., and Rao, A. V., "Pseudospectral Methods for Solving Infinite-Horizon Optimal Control Problems," Automatica, Vol. 47, No. 4, April 2011, pp. 829-837. doi:10.1016/j.automatica.2011.01.085

[19] Gong, Q., Kang, W., and Ross, I. M., "A Pseudospectral Method for the Optimal Control of Feedback Linearizable Systems," IEEE Transactions on Automatic Control, Vol. 51, No. 7, July 2006, pp. 11151129. doi: $\underline{10.1109 / \text { TAC.2006.878570 }}$ 
[20] Hager, W. W., "Runge-Kutta Methods in Optimal Control and the Transformed Adjoint System," Numerische Mathematik, Vol. 87, No. 2, Dec. 2000 , pp. $247-282$.

doi: $10.1007 / \mathrm{s} 002110000178$

[21] Abramowitz, M., and Stegun, I., Handbook of Mathematical Functions with Formulas, Graphs, and Mathematical Tables, Dover, New York, 1965, pp. 887-888.

[22] Kirk, D. E., Optimal Control Theory: An Introduction, Dover, Mineola, NY, 2004, pp. 184-325.
[23] Bryson, A. E., and Ho, Y.-C., Applied Optimal Control, Hemisphere Publ., New York, 1975, pp. 121-123.

[24] Gill, P. E., Murray, W., and Saunders, M. A., SNOPT: An SQP Algorithm for Large-Scale Constrained Optimization, SIAM Review, Vol. 47, No. 1, Jan.-March 2005, pp. 99-131.

D. Spencer Associate Editor 